\title{
MEASUREMENTS OF RESIDUAL RADIOACTIVITY IN NEUTRON TRANSMUTATION DOPED THERMISTORS
}

\author{
A. Alessandrello, C. Brofferio, D.V. Camin, O. Cremonesi, E. Fiorini, A. Giuliani, M. \\ Pavan, G. Pessina, E. Previtali, and L. Zanotti \\ Dipartimento di Fisica dell' Universita' di Milano and Sezione di Milano dell' INFN, \\ Milano I-20133, Italy
}

\begin{abstract}
Germanium wafers exposed to intense neutron beams from a nuclear reactor to produce Neutron Transmutation Doped thermistors have been analysed for residual radioactivity in view of their application in experiments on rare decays. Measurements have been routinely carried out for more than three years with Germanium spectrometers of low intrinsic radioactivity operating in the Gran Sasso Underground Laboratory in order to suppress the background due to cosmic rays. Four long living nuclei produced during the irradiation by the fast neutron component of the flux have been identified. It is shown that two of them are going to contribute considerably to the background in experiments on rare events to be carried out with thermal detectors, especially in the direct search of dark matter. These measurements enable also to investigate the presence of contaminants in the germanium wafer before the irradiation. The sensitivity can be as low as $10^{-14} \mathrm{~g} / \mathrm{g}$ for some elements.
\end{abstract}

Milan, March 11, 1994

Submitted to Nucl.Instrum.and Meth. B

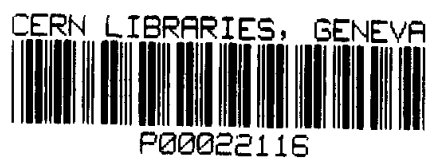


包

Counts

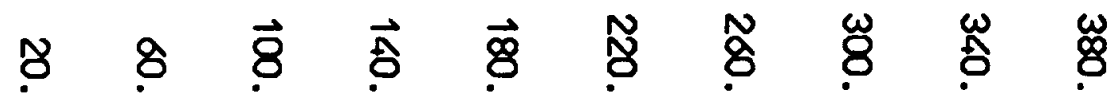

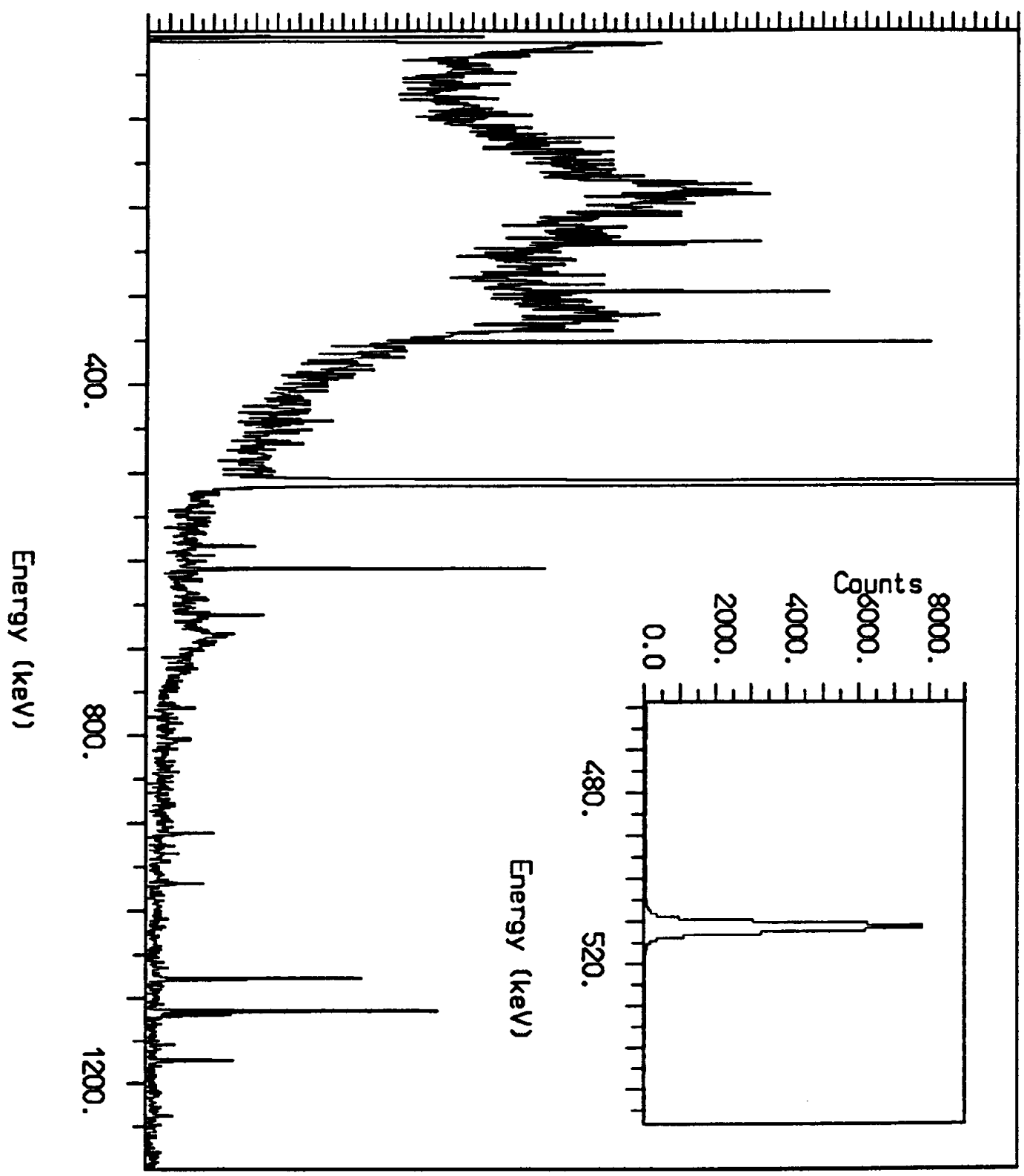




\section{Introduction}

Considerable interest has been addressed recently to the use of thermal detectors in searches on rare events like double beta decay [1] and interactions of dark matter particles [2] or solar neutrinos [3,4]. Experiments are planned or are already in operation $[5,6]$ where particles are detected by bolometers made with a large crystal, usually diamagnetic and dielectric, and with a thermometer in contact with it. The heat capacity of the crystal, when operated at temperatures of a few tens of millikelvin, is so low that even the tiny energy delivered by an incident or internally generated particle can induce a measurable increase of temperature. In most cases the thermometer is a Neutron Transmutation Doped (NTD) thermistor obtained with an intense irradiation of ultrapure germanium with neutrons from a nuclear reactor [7]. This technique produces a very uniform and reproducible density of acceptors and donors.

Thermal neutron capture on ${ }^{70} \mathrm{Ge}$ (20.5\% isotopic aboundance) produces $71_{\mathrm{Ge}}$ which decays with a lifetime of 11.4 days in the acceptor $\left(71_{\mathrm{Ga}}\right)$. On the contrary thermal capture on ${ }^{74} \mathrm{Ge}\left(36.5 \%\right.$ i.a.) leads to ${ }^{75} \mathrm{Ge}$ which $\beta$-decays with a lifetime of 1.38 hours into the donor $\left({ }^{75} \mathrm{As}\right)$. Neutron capture occurs also on $76 \mathrm{Ge}$ (7.6\% i.a.) leading to ${ }^{77} \mathrm{Ge}$ with a consequent decay (11.3 hours) into ${ }^{77} \mathrm{As}$, which however is unstable and produces ( 38.8 hours) the "double donor" $77 \mathrm{Se}$. NTD germanium has therefore both types of dopants and is autocompensated, unlike Si and GaAs NTD thermistors. Recently however NTD thermistors containing only pdopants have been obtained by irradiating a ${ }^{70} \mathrm{Ge}$ isotopically enriched crystal [8].

Experiments on rare events are normally carried out underground to suppress the background due to cosmic rays and are heavily shielded against local radioactivity. In addition, any sizable source of internal radioactive contamination has to be eliminated [9]. This problem has never been investigated in the planned use of bolometers employing NTD thermistors, since all captures by thermal neutrons in germanium lead to short living radioactive nuclei whose contribution to background becomes negligible a few months after irradiation. We were however worried by the possible presence of long living radioactive nuclides produced by interactions of the fast neutron component of the reactor beam. It is in fact known that when a very intense irradiation is needed in a reactor the fast component in the neutron beam can be as high as a few percent. In addition long living radioactive isotopes can be produced by capture of thermal neutrons on some impurity existing in the germanium wafer.

We report here the results of systematic measurements carried out over more than three years in the Gran Sasso Laboratory on the residual activity of a Germanium sample irradiated for the production of NTD thermistors. 


\section{Experimental details}

The measurements have been carried out on three wafers of Neutron Transmutation Doped Germanium totalling 30.2 grams, which were exposed in the period from middle of October to middle of December 1990 to fluences ranging from $3.23 \times 10^{18}$ to $3.4910^{18} \mathrm{n} \mathrm{cm}^{-2}$ at the University of Missouri Research Reactor. A first series of four measurements on the $\gamma$-activity of these wafers was carried out at average times of $100,122,149$ and 190 days after irradiation. The detector was a GeLi detector with $30 \%$ efficiency relative to $\mathrm{NaI}$, shielded with low radioactivity lead and Oxygen Free High Conductivity Copper with a minimum thickness of 5 and $15 \mathrm{~cm}$, respectively. The experimental set-up was operated at a depth of 3500 metres of water equivalent in the Gran Sasso Underground Laboratory in order to suppress the background due to cosmic ray particles. In particular muons and neutrons are reduced by six and three orders of magnitude with respect to surface [10].The activity of the irradiated sample was considerable and produced in the germanium spectrum a large bump below $300 \mathrm{keV}$ approximately, clearly indicating bremsstrahlung from beta active nuclei produced by the irradiation. Due to this bump only $\gamma$-lines above this energy region could be detected unambigously in these early measurements. Further measurements were carried out at average times of 438, 732 and 1127 days after exposure, with an intrinsic Germanium diode of $86 \%$ relative efficiency. All the spectrum could be investigated in these runs.

\section{Results and interpretation}

In addition to the peaks due to natural radioactive background, $13 \gamma$-lines due to neutron activation were found (fig.1). They are reported in Table I. All these lines can be unambigously attributed to the presence of 5 radioactive contaminants on the basis of their energy, relative intensity and dependence on time after exposure. The lifetimes measured in this experiment are in good agreement with those reported in the literature [11].

We would like to stress that only one of these nuclei was produced by thermal neutron capture. To us the most likely interpretations are:

a. ${ }^{75} \mathrm{Se}$, which decays by electron capture, has been produced by $\alpha$-particles from the $(n, \alpha)$ reactions induced on germanium by fast neutrons during the irradiation. Possible interactions are:

${ }^{72} \mathrm{Ge}\left(27.4 \%\right.$ isotopic aboundance) $(\alpha, \mathrm{n}){ }^{75} \mathrm{Se}$

or (less likely)

${ }^{73} \mathrm{Ge}\left(7.8 \%\right.$ i. a.) $(\alpha, 2 \mathrm{n}){ }^{75} \mathrm{Se}$ and ${ }^{74} \mathrm{Ge}\left(36.5 \%\right.$ i. a.) $(\alpha, 3 \mathrm{n}){ }^{75} \mathrm{Se}$. 
With a weighted average on the values extracted for the various peaks we extrapolate an initial contamination of $3.3 \pm .8 \mathrm{~Bq} \mathrm{~kg}^{-1}$ due to this nucleus. This activity becomes small (less than $10 \mathrm{mBq} \mathrm{kg}^{-1}$ ) after three years.

b. ${ }^{74} \mathrm{As}$, which decays by electron capture and positron emission, has been produced by protons from $(p, n)$ reactions on germanium during irradiation. Possible interactions are:

${ }^{74} \mathrm{Ge}(36.5 \%$ i. a. $)(\mathrm{p}, \mathrm{n}){ }^{74} \mathrm{As}$

or, less likely:

${ }^{73} \mathrm{Ge}\left(36.5 \%\right.$ i. a.) $(\mathrm{n}, \gamma){ }^{74} \mathrm{As}$.

The average contamination due to this nucleus is considerable immediately after irradiation $\left(4400 \pm 800 \mathrm{~Bq} \mathrm{~kg}^{-1}\right)$, but becomes irrelevant after three years.

c. ${ }^{68} \mathrm{Ge}$ decays by electron capture to ${ }^{68} \mathrm{Ga}$, with a further decay of this nucleus by positron emission and electron capture $(1.130 \mathrm{~h}$ lifetime). It was produced during irradiation by fast neutrons via the reaction:

${ }^{70} \mathrm{Ge}(20.5 \%$ i. a. $)(\mathrm{n}, 3 \mathrm{n}){ }^{68} \mathrm{Ge}$

or, less likely,

${ }^{72} \mathrm{Ge}\left(27.4 \%\right.$ i. a.) $(\mathrm{n}, 5 \mathrm{n}){ }^{68} \mathrm{Ge}$.

The initial contamination by this nucleus was determined using the intense 511 annihilation $\gamma$-line due to annihilation of the positron emitted by ${ }^{68} \mathrm{Ga}$. The contribution of positrons from ${ }^{74} \mathrm{As}$, which is however relevant only in the first period after irradiation, is subtracted. The initial contamination due to ${ }^{68} \mathrm{Ge}$ obtained from the $511 \mathrm{keV}$ line $\left(160 \pm 2 \mathrm{~Bq} \mathrm{~kg}^{-1}\right)$ is in excellent agreement with the value extracted from the 1077 line $\left(150 \pm 10 \mathrm{~Bq} \mathrm{~kg}^{-1}\right.$.). This contamination is still considerable after three years (about $10 \mathrm{~Bq} \mathrm{~kg}^{-1}$ ).

d. ${ }^{65} \mathrm{Zn}$, which decays by electron capture, was mainly produced during irradiation by fast neutrons via the reaction:

${ }^{70} \mathrm{Ge}(20.5 \%$ i. a.) $(\mathrm{n}, \alpha 2 \mathrm{n}) 65 \mathrm{Zn}$

Activities following immediately irradiation and three years after are ( $15 \pm 2$ $\mathrm{Bq} \mathrm{kg}^{-1}$ ) and $\sim .7 \mathrm{~Bq} \mathrm{~kg}^{-1}$, respectively. 
e. ${ }^{95} \mathrm{Zr} \beta$-decays to $95 \mathrm{Nb}$ with the emission of two $\gamma$-lines at 724 and 757 $\mathrm{keV}$. A further $\beta$-decay of ${ }^{95} \mathrm{Nb}$ however occurs with a lifetime of 34.97 days and the emission of a $\gamma$-line at $765 \mathrm{keV}$.

Activities immediately after irradiation and three years after are $(50 \pm 10 \mathrm{~Bq}$ $\mathrm{kg}^{-1}$ ) and less than a mBq kg-1, respectively. The origin of this contamination is unclear. It could be due to activation by thermal neutrons of some impurity of ${ }^{94} \mathrm{Zr}$, which is normally present in mechanic tools. Taking into account the capture cross section, the isotopic aboundance of $94 \mathrm{Zr}$ and the neutron flux, a very tiny impurity ( less than $10^{-10} \mathrm{gram} / \mathrm{gram}$ of natural Zirconium in the Germanium wafer) would account for this contamination.

\section{Conclusions}

Our measurements show that after a few months from the irradiation contaminatios from ${ }^{75} \mathrm{Se},{ }^{74} \mathrm{As},{ }^{75} \mathrm{Zn}$ and ${ }^{68} \mathrm{Ge}$ due to interactions induced by fast neutrons are present. Even after three years the contaminations from $65 \mathrm{Zn}$ and ${ }^{68} \mathrm{Ge}$ are considerable : of the order of about one and ten $\mathrm{Bq} \mathrm{kg}^{-1}$, respectively. One could note that in thermal detectors pulses generated inside the thermistor have a much faster rise-time than those from the crystal and can therefore be mostly eliminated by a suitable pulse shape analysis. Both nuclei however decay by electron capture and the emitted $\mathrm{X}$-rays and Auger electrons could considerably contribute to background in the low energy region which is relevant in searches for direct interactions of dark matter particles.

The intense irradiation needed to produce NTD thermistors requires severe constraints on the purity of the Germanium wafers. In fact our measurements allow a precise analysis on the purity of the target Germanium, based on activation of long living nuclei. The results are shown in Table II. One can see that the only detectable activity is that induced by activation of ${ }^{94} \mathrm{Zr}$, which becomes however negligible with time.

In addition to the above mentioned application to thermal detectors, artificial neutron activation can be performed in view of other experiments on rare decays. It can help for instance in the study of the internal contamination of Germanium diodes due to ${ }^{68} \mathrm{Ge}$ and ${ }^{65} \mathrm{Zn}$ cosmogenically produced by atmosheric neutrons [9] or to ${ }^{73} \mathrm{As},{ }^{74} \mathrm{As}$ and ${ }^{75} \mathrm{Se}$ produced by neutron generated protons and $\alpha$-particles, respectively. With this aim we are exposing to fast neutrons samples of $\mathrm{TeO}_{2}$ to investigate the cosmogenic internal background of thermal $\mathrm{TeO}_{2}$ detectors which we are using in a double beta experiment on ${ }^{130} \mathrm{Te}$ [12]. Exposures are also being carried out on Copper to investigate the background due to interactions of atmospheric neutrons on this material, commonly used for shielding experiments on rare decays. 


\section{Aknowledgments}

We would like to express our gratitude to Eugene Haller and his group for production and continuous collaboration on the operation of Germanium NTD thermistors, and to Cristina Arpesella and Paolo Sverzellati for generous help in the course of these measurements. 
[1] M.K.Moe, Int.J.Mod.Phys. E2:507 (1993); M.K.Moe and P.Vogel, Submitted to Annual Review of Nuclear and Particle Sciences; T.Tomoda, Rep.Prog. Phys. 54 (1991) 53; H.V.Klapdor, J.Phys.G: Nucl.Part.Phys. 17 (1991) S129; E.Fiorini , Nucl.Phys.News. 1 (1991) 17

[2] P.F.Smith and J.D.Lewin, Phys.Rep. 187 (1990) 203, and B.Sadoulet , invited paper to TAUP93, Gran Sasso 19-23 Sept.1993, ed.by A.Bottino, to be published in Nucl.Phys.B

[3] J.N.Bahcall, Neutrino Astrophysics, Cambridge Univ.Press, 1989, and previous references therein;

[4] See for instance Proc.of the V Intern.Workshop on "Neutrino Telescopes", Venice March 2-4 1993, ed.by M.Baldo Ceolin;

[5] E.Fiorini: Physical motivations for thermal detectors, J. Low Temp. Phys. 93 (1993) 189;

[6] For more details on thermal detectors see :Workshop on Low Temperature Detectors for Neutrinos and Dark Matter - Ringberg , March 1987, ed.by K.Pretzl (Springer 1987); II Europ.Workshop on Low Temperature Devices for the Detection of Low Energy Neutrinos and Dark Matter- LAPP (Annecy), May 1988, eds. L.Gonzales Mestres, and D.Perret Gallix (Editions Frontieres 1989); III Int.Work. on Low Temperature neutrinos and Dark Matter- Gran Sasso, September 1989, eds. L.Brogiato, D.Camin and E.Fiorini (Editions Frontieres 1990); Workshop on Low Temperature Detectors for Neutrinos and Dark Matter IVOxford, September 1991, eds. N.E.Both and G.Salmon (Editions Frontieres 1992); V Int.Workshop on Low Temperature Detectors- Berkeley, July 1993 eds. B.Young and S.Labov, J of Low Temp.Phys. Vol.95 (1993)

[7] E.E. Haller, N.P. Palaio, M. Rodder, W.L.Hansen, and E.Kreisa, in Neutron Transmutation Doping of Semiconductor Materials, ed. by R.D. Larrabee (Plenum, New York 1984), p.21

[8] K. M. Itoh et al, Jour.of Low Temp.Phys., 93 (1993) 307

[9] G. Heusser, Nucl.Instrum. and Meth. B 83 (1993) 223

[10] L. Zanotti, J.Phys. G17 (1991) S373

[11] See for instance: E.Browne and R.B.Firestone, Tables of Radioactive Isotopes, Ed. V.S.Shirley, Johm Wiley and Sons (1986) [12] A. Alessandrello et al, Submitted to Phys.Lett.B 
T A B L E I : Radioactive contaminants

\begin{tabular}{|c|c|c|c|c|c|}
\hline Nucleus & ${ }^{75} \mathrm{Se}$ & ${ }^{74}$ As & ${ }^{65} \mathrm{Zn}$ & ${ }^{68} \mathbf{G e}$ & $95 \mathbf{Z r}$ \\
\hline Measured & 121 & 596 & 1115 & 511 & 724 \\
\hline Lines (kev) & $\begin{array}{l}136 \\
265 \\
280 \\
401\end{array}$ & 635 & & 1077 & $\begin{array}{c}754 \\
765(95 \mathrm{Nl}\end{array}$ \\
\hline $\begin{array}{l}\text { Initial activity } \\
(\mathrm{Bq} / \mathrm{kg})\end{array}$ & $3.3 \pm .8$ & $4400 \pm 750$ & $15 \pm 2$ & \multicolumn{2}{|c|}{$150 \pm 10(1077$ line $)$} \\
\hline \multicolumn{6}{|l|}{ Lifetime (d) } \\
\hline Measured & $145 \pm 17$ & $18 \pm 6$ & $253 \pm 8$ & \multicolumn{2}{|c|}{$272 \pm 12(1077$ line $)$} \\
\hline Literature & 119.8 & 17.8 & 243.8 & 270.8 & 64.02 \\
\hline
\end{tabular}


T A B L E II : Elemental contaminations in Germanium wafers (pg/g)

\begin{tabular}{|c|c|c|c|c|c|}
\hline Sc & $<0.05$ & $\mathrm{Cr}$ & $<1000$ & Co & $<1$ \\
\hline $\mathrm{Zn}$ & $<10$ & $\mathrm{Se}$ & $<10$ & $\mathrm{Rb}$ & $<1000$ \\
\hline $\mathbf{Z r}$ & $\sim 100$ & $\mathrm{Ru}$ & $<10$ & $\mathrm{Ag}$ & $<1$ \\
\hline In & $<10$ & Sn & $<1000$ & $\mathrm{Sb}$ & $<1$ \\
\hline $\mathrm{Te}$ & $<100$ & Cs & $<0.1$ & $\mathrm{Ce}$ & $<50$ \\
\hline $\mathrm{Nd}$ & $<1000$ & $\mathrm{Sm}$ & $<100$ & $\mathrm{Gd}$ & $<10$ \\
\hline $\mathrm{Tb}$ & $<0.1$ & Dy & $<10$ & $\mathrm{Tm}$ & $<.1$ \\
\hline $\mathrm{Yb}$ & $<.1$ & $\mathrm{Lu}$ & $<100$ & Hf & $<10$ \\
\hline $\mathrm{Ta}$ & $<.1$ & Os & $<100$ & Ir & $<.01$ \\
\hline & $\mathrm{Hg}$ & $<10$ & & & \\
\hline
\end{tabular}

\section{F I G UR E CAPT I O N}

Fig. 1 : Remaining activity in the germanium wafers three years after irradiation (measurement totalling 300 hours of effective running time). The intense peak at $511 \mathrm{keV}$ is shown in the inset. 